\title{
An Intelligent Agents-Based Virtually Defaultless Check System: The SafeCheck System
}

\author{
Jae Kyu Lee and Han Seong Yoon
}

ABSTRACT: The conventional system of paper checkbooks with multiple checks carries a significant risk of default because no form of authorization is required when individual checks are written, although implicitly each checkbook is authorized when it is issued to the payer. Nonetheless, checks are a less expensive payment method than credit cards, so they are suitable for a high-credit business environment. To make up for the defect in the paper check system, whereby checks likely to default can be issued without authorization, the authors have devised an agent-based electronic check system known as SafeCheck that can monitor the situation and block the issuance of nonallowable checks in a distributed manner. Three types of service are allowed, depending upon the check issuer's credibility. Members of the top credit class require authorization only for each checkbook. Members of the second-level credit class require authorization for each check. Members of the thirdlevel credit class are allowed to issue checks only within their checking account balance. The bank can dynamically adjust the credit level depending upon the record of defaults. The SafeCheck System consists of three agents: Checkbook Agent at the check issuer's site, Check-Receipt Agent at the check receiver's site, and the bank's Control Agents at the check issuer's and receiver's banks respectively. For security purposes, SafeCheck has public key cryptography, digital signatures, and certificate schemes like those of the SET protocol for credit cards. The essence of a checkbook agent can be stored in the IC card.

KEY WORDS AND PHRASES: Check systems, default risk, electronic payments, intelligent agents, security.

With the rapid expansion of electronic commerce on the Internet [15, 24, 33], electronic payment systems have become more and more necessary. Several researchers have been seeking to develop secure, efficient electronic payment systems $[5,24,34]$. Currently, the most popular payment methods for consumer electronic ordering on the Internet are credit cards, electronic fund transfers, and electronic checks. Security is a major concern at this stage, and the Secure Electronic Transfer (SET) protocol is gaining in popularity [28]. Nevertheless, paper checks are still popular in the United States for business and personal payments, as shown in Figure 1, and the total amount of payments by check is growing [19]. As the proportion of business-to-business e-commerce increases, the electronic check system will become more important.

There have been several attempts to build an electronic version of the paper check system. However, electronic versions of a paper check system turn out to be very similar to electronic fund transfer (EFT) or credit card systems. The electronic check system resembles EFT because the payee's presentation of checks to its bank and check-clearing requests by the payee's bank to the payer's bank can be executed electronically almost at the same time the check is issued. Thus it is no longer possible to issue overdraft checks, which is bad news from the standpoint of the payer's cash flow. If the clearing date is institutionally fixed to keep the benefit of credit, electronic checks become more similar to credit 


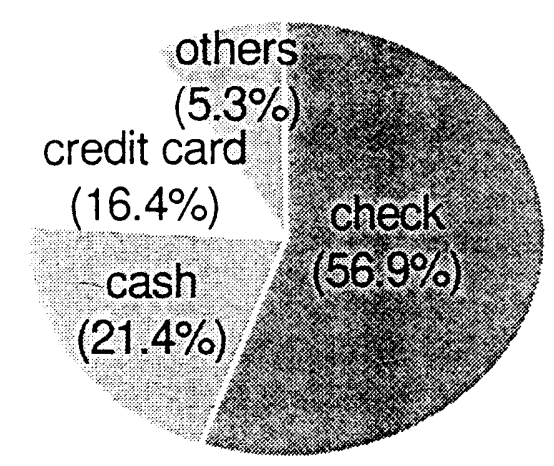

Figure 1. Consumer Spending Ratio in United States in 1996 [19]

Source: Green Sheet Online (1997)

cards. However, one fundamental difference pertains to the frequency of payment authorization. While the credit card system requires an authorization for each payment transaction, check payment systems give the authorization at the checkbook level, following the customary practice of paper check systems. This means that the check system requires less authorization effort, but at the risk of default.

In a paper check system, the payee cannot verify the balance in the payer's checking account, as would be necessary to estimate the risk of default, and the payer's bank is not aware that a bad check has been issued until it is returned from the payee's side. The only way for a payee to completely avoid the risk of default is to accept nothing but certified checks, but this restriction would introduce an element of rigidity into the transaction.

The good news with the electronic check system is that the payee's bank and the payer's bank can both control the relevant information in the payer's electronic checkbook in a distributed manner to avoid abuses. They can do this by adopting an electronic checkbook agent that has dynamic self-control and the capability to make timely inquiries to the control agents at other banks. Distributed control capability of this kind can reduce the risk of default in advance without an astronomical explosion of authorization effort. By significantly reducing the risk of default, this system makes it feasible to design a check system that is free of default risk at little or no extra cost to the payers. Since most defaults can be avoided in advance, any risk penalty charged to the payers will be affordable. In some instances the payer bank will elect to bear the default losses in compensation for the increased revenue owing to the payee's preference. Depending on the bank's managerial policy, several different administrative methods can be used to compensate for the minor default losses inherent on the payer's bank's side.

This paper describes an electronic check system that has the main advantage of paper checks (i.e., it allows payers to write overdrafts within a designated limit) and at the same time reduces or eliminates their disadvantages (i.e., it virtually eliminates the risk of default for the payee), and has a lower authorization cost than the credit card system, which requires authorization for every payment. This new electronic payment system is default-risk-free for payees and more economical than credit card systems. For these reasons it should be acceptable both to high-credit individuals and to the business community. 
The proposed electronic check system is called SafeCheck. It consists of three agents: Checkbook Agent at the payer's site, Check-Receipt Agent at the payee's site, and Bank's Control Agent at the payer's and payee's bank sites.

\section{Types of Electronic Payment Systems}

\section{Classification of Electronic Payment Systems}

Four electronic payment systems are currently available on the Internet: electronic cash, electronic fund transfers, credit cards, and electronic checks. The main differences between these systems pertain to anonymity of payer and payee, level of default risk to payee, permission of credit to payer, and issuance authorization frequency (see Table 1).

\section{Electronic Cash}

In most electronic cash systems, the electronic cash can be transferred from the payer's credit card or checking account to the payer's electronic wallet, and vice versa. The received electronic cash has the value of real cash and there is no risk of default. CyberCoin provides a service of this kind on the Internet [7]. Most IC card-based electronic wallets, such as VisaCash, adopt a "closed system" that allows the transfer of money in the IC card only to and from the bank's account, but so far the network for transmission is not the Internet, although there has been some movement toward using it [48]. Mondex has adopted an "open system" that allows direct transfers between electronic wallets without intervention of the bank's account [30].

\section{Electronic Fund Transfers}

Electronic Fund Transfer (EFT) has been a popular service for several decades on the non-Internet network and is now being attempted on the Internet. The QuickPay system of Security First Network Bank (SFNB) can be classified as an Internet-based electronic fund transfer service [40]. QuickPay also has a complementary paper check feature in case the payee does not have an electronic facility. However, its certified checks are different from regular checks in that the amount is deducted from the payer's account at the time of check issuance.

\section{Credit Cards}

Credit cards employing the Secure Socket Layer encryption scheme are the most popular form of electronic payments for cybershopping [32]. Since the current scheme is not safe from masquerading users, the SET (Secure Electronic Transaction) protocol, which is equipped with public key encryption as well as certificates, has been proposed by Visa and MasterCard and is gaining in popularity [28]. 


\begin{tabular}{|c|c|c|c|c|c|}
\hline Attributes & $\begin{array}{c}\text { Electronic } \\
\text { cash }\end{array}$ & $\begin{array}{c}\text { Electronic } \\
\text { fund } \\
\text { transfer }\end{array}$ & $\begin{array}{l}\text { Credit } \\
\text { card }\end{array}$ & $\begin{array}{c}\text { Electronic } \\
\text { check }\end{array}$ & $\begin{array}{c}\text { Agent-based } \\
\text { check: } \\
\text { SafeCheck }\end{array}$ \\
\hline $\begin{array}{l}\text { Anonymity of } \\
\text { payer and } \\
\text { payee }\end{array}$ & Anonymous & Anonymous & Anonymous & Anonymous & Anonymous \\
\hline $\begin{array}{c}\text { Default risk to } \\
\text { payee }\end{array}$ & Risk free & Risk free & Risk free & $\begin{array}{l}\text { Risky (unless } \\
\text { certified check) }\end{array}$ & $\begin{array}{l}\text { Virtually risk- } \\
\text { free with } \\
\text { complementary } \\
\text { administrative } \\
\text { treatment }\end{array}$ \\
\hline $\begin{array}{c}\text { Permission of } \\
\text { credit }\end{array}$ & Not allowed & Not allowed & Allowed & Allowed & Allowed \\
\hline $\begin{array}{c}\text { Issuance } \\
\text { authorization } \\
\text { frequency }\end{array}$ & $\begin{array}{c}\text { Self-check the } \\
\text { availability of } \\
\text { cash in wallet } \\
\text { for every } \\
\text { payment }\end{array}$ & $\begin{array}{c}\text { Once for every } \\
\text { payment }\end{array}$ & $\begin{array}{c}\text { Once for every } \\
\text { payment }\end{array}$ & $\begin{array}{l}\text { In principle, } \\
\text { once for every } \\
\text { checkbook lor } \\
\text { once for every } \\
\text { check issuancel }\end{array}$ & $\begin{array}{l}\text { Once for every } \\
\text { checkbook if } \\
\text { credible (level } \\
\text { of credibility } \\
\text { can be } \\
\text { dynamically } \\
\text { adjusted) }\end{array}$ \\
\hline $\begin{array}{l}\text { Available } \\
\text { systems }\end{array}$ & $\begin{array}{l}\text { DigiCash's } \\
\text { eCash [10], } \\
\text { NetCash [29], } \\
\text { CyberCoin [7], } \\
\text { Mondex [30], } \\
\text { VisaCash [48] }\end{array}$ & $\begin{array}{c}\text { SFNB's } \\
\text { QuickPay }[40]\end{array}$ & $\begin{array}{c}\text { CyberCash [8], } \\
\text { SET protocol } \\
{[28]}\end{array}$ & $\begin{array}{c}\text { FSTC's } \\
\text { Electronic Check } \\
\text { [17], NetCheque } \\
\text { [34], VirtualPin } \\
{[13], \text { NetBill [4], }} \\
\text { PayNow [8], } \\
\text { NetChex [31] }\end{array}$ & SafeCheck \\
\hline
\end{tabular}

Table 1. Types of Electronic Payment Systems.

\section{Electronic Checks}

The electronic check replicates the ordinary paper check. The Financial Services Technology Consortium (FSTC) employs encryption, certification, and IC identification cards to deal with the security issue, and has proposed four functional-flow scenarios, as depicted in Figure 2 [17]. The "Deposit and Clear Scenario" and "Cash and Transfer Scenario," both of which include a clearing process, are not completely safe from the risk of default. The other two scenarios are actually variant forms of electronic fund transfer, although they are classified as electronic checks. This illustrates the phenomenon, mentioned earlier, of merging electronic checks with electronic fund transfers. Note that none of the scenarios give sufficient attention to the reduction of authorization frequency. First Virtual's VirtualPin takes an idiosyncratic variation from other traditional electronic check systems [13]. It automatically withdraws the issued amount from the payer's account at the time the payer approves and deposits the withdrawn amount to the payee's account. 


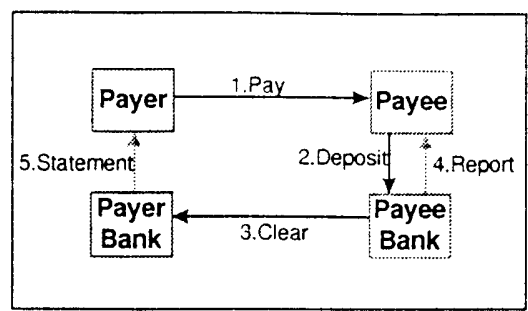

(a) The Deposit and Clear Scenario

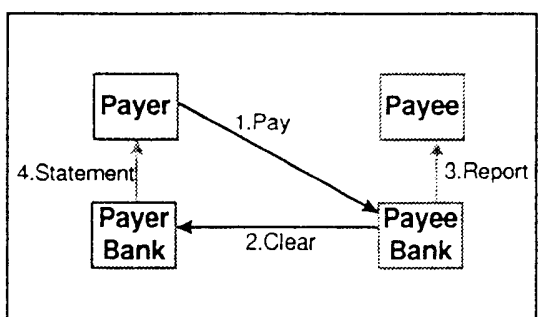

(c) The Lockbox Scenario

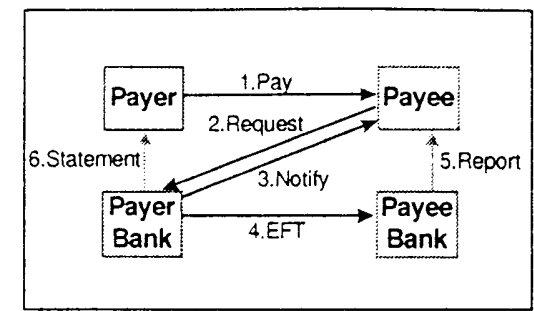

(b) The Cash and Transfer Scenario

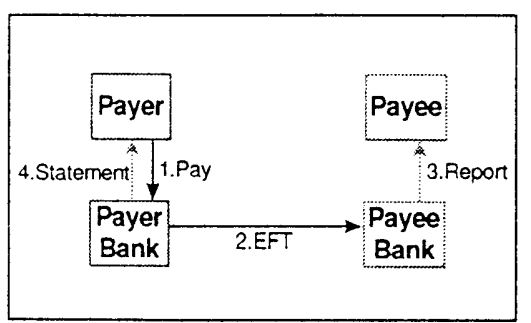

(d) The Funds Transfer Scenario

Figure 2. Scenarios of Functional Flows for Electronic Checks [17]

\section{SafeCheck System}

As can be seen from Table 1, the agent-based SafeCheck System proposed here is a new type of electronic payment system that avoids (or at least reduces) the default risk on the payee's side, reduces the cost of authorization, and permits credit-based payments. As mentioned earlier, the SafeCheck System consists of three types of agents: Checkbook, Check-Receipt, and Bank's Control Agents, as depicted in Figure 3. The SafeCheck System is basically the same as "Deposit and Clear" in the FSTC classification.

\section{Variations of Checks}

There are several variations on the four typical electronic payment systems. The certified check and the crossed check are variations on the regular check. Certified checks are similar to regular checks except that within certain limits their overdrafts have to be certified by the check issuer's (payer's) bank. The grounds for certification may be a mortgage or the issuer's high credit level. Crossed checks carry a particular restriction on the receiving banks or purchasable goods. Note that a centralized clearing institution is needed, as is also essential for efficient exchanges of paper checks among multiple banks. However, a centralized clearing service may not be necessary in an electronic check environment if the associated banks can transfer the clearing amounts bilaterally on-line.

\section{Architecture of Agents for SafeCheck}

While definitions of the intelligent agent differ somewhat [16, 18, 47], all agree that its key attributes are autonomy, communication ability, and reasoning and learning capability $[16,35]$. Intelligent agents are applicable to financial deci- 


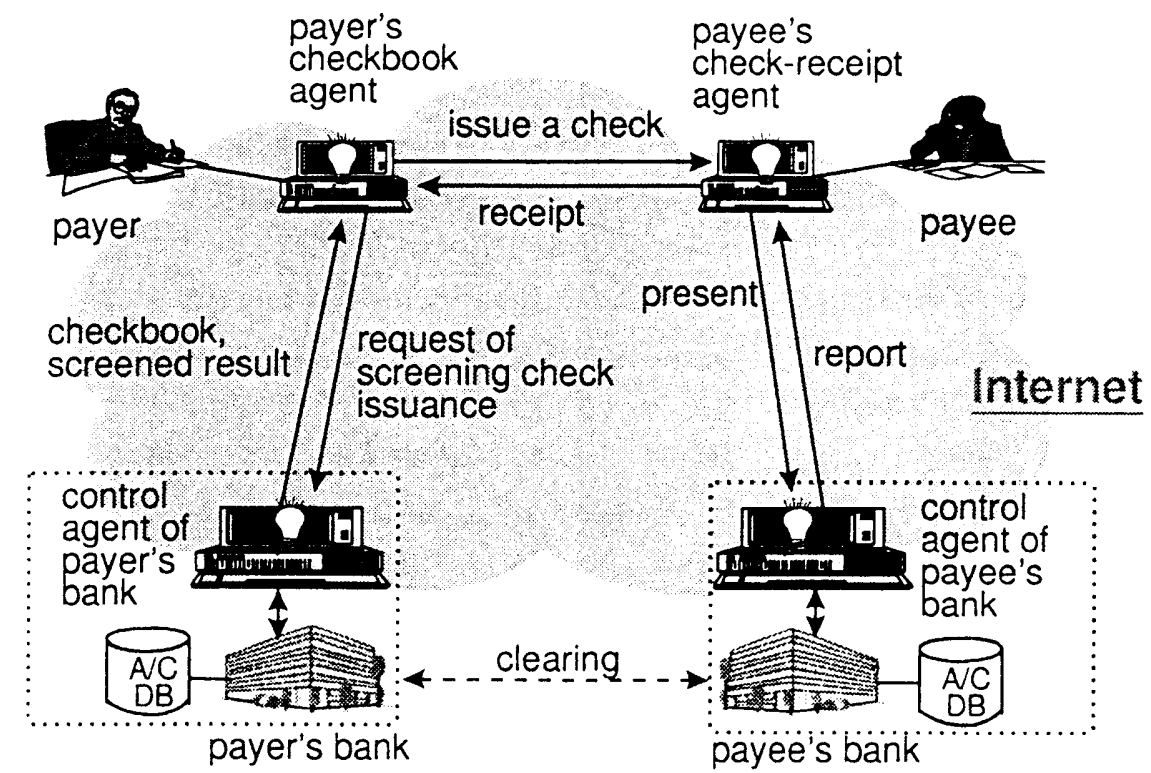

Figure 3. SafeCheck System Architecture

sions related to personal banking, electronic payments, budgeting, and so on [37]. Several capabilities of intelligent agents are applied to banking and payments in the Minnesota Agent Marketplace Architecture (MAGMA) [44] and the Practical Interactive and Personizable MARkeTplace (PIPEMART) [22]. These systems map the existing electronic payment procedure to the communication between agents.

In the SafeCheck system, each intelligent agent (SafeCheck Agent) consists of a problem solver and a communication controller. In contrast to traditional expert systems with a single inference (or solution) engine, a SafeCheck Agent has a problem-solving manager and a communication controller, as depicted in Figure 4. SafeCheck Agents utilize the concept of UNIK-AGENT [27]. Although the SafeCheck Agents for the various participants (payer, payee, bank) have basically the same architecture, the bank's control agent is the most complex, and has the largest amount of knowledge and data.

\section{Problem Solver}

The problem-solving manager and the solution engines are the main components of the problem solver. The problem-solving manager selects an adequate solution engine based on the context and the messages received from human or other agents. Solution engines are a collection of problem-solving methods, such as data retrieval, knowled ge-based reasoning, neural network-based estimation or classification, specific functions, and outgoing-message generation. Agents may be equipped with different solution engines depending upon their roles. Thus, a domain-specific analysis for the design of solution engines and messages is essential in the first place. Nevertheless, the agent should maintain a standard architecture so as to be compatible with other solution engines, messages, and data. 


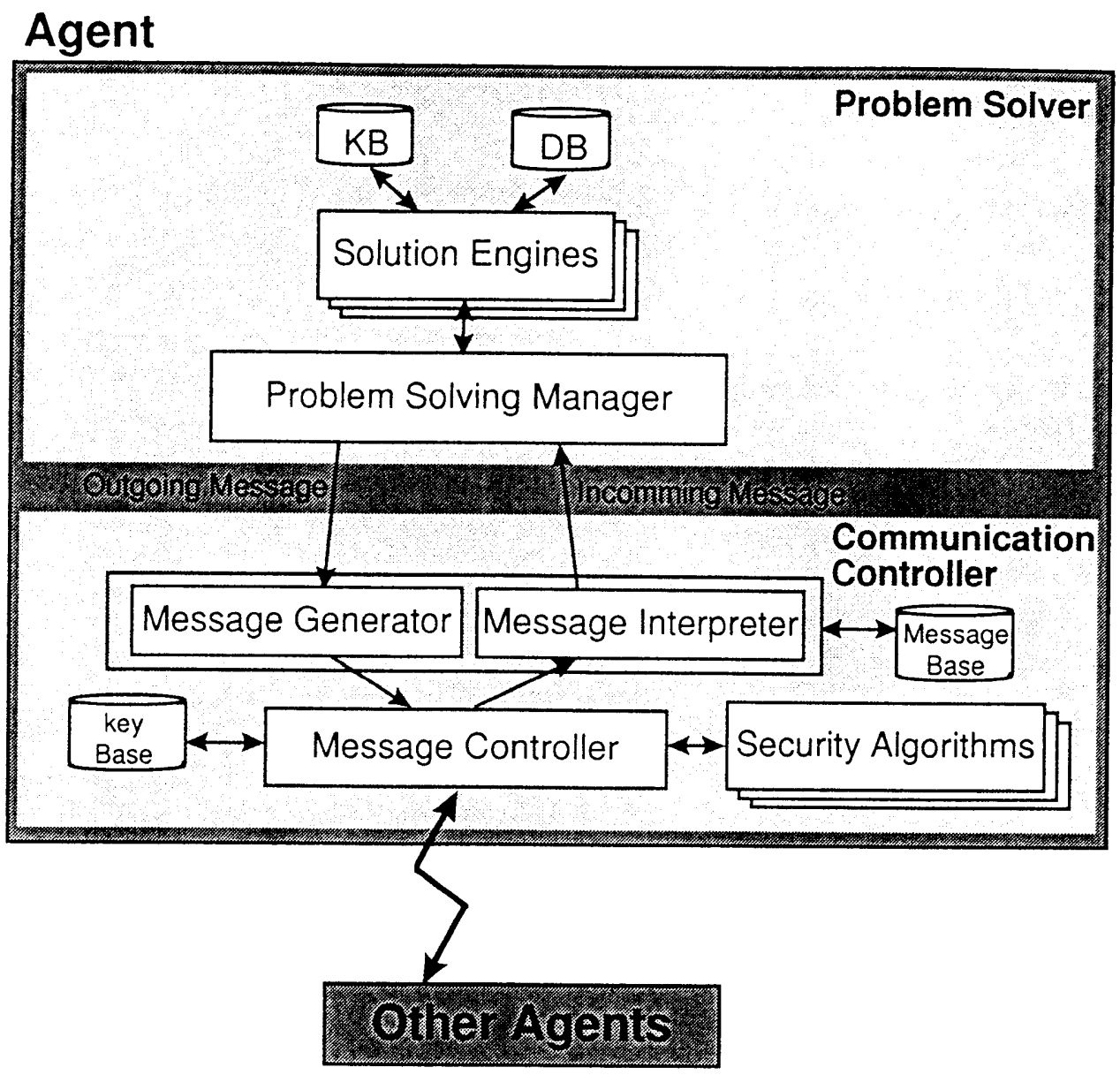

Figure 4. Architecture of SafeCheck Agent

For instance, for a bank's control agent, Figure 5 shows a neural network model adopted to the credit-evaluation model. The adequacy of neural network models for credit evaluation has been empirically studied [1, 36, 43, 46]. The incoming message to the agent should include input data to this model, and the generated outgoing message must have utilized the output from the model. On the other hand, for a checkbook agent, a rule-based system along with functions is adopted for decisions concerning the issuance of overdraft checks $[3,38,41]$. In this manner, the key roles, the solution engines, and the origin and destination of each agent's messages are designed as in Table 2.

\section{Communication Controller}

The communication controller consists of an Incoming Message Interpreter, Outgoing Message Generator, Message Controller, and Security Algorithms. The message interpreter interprets and validates incoming messages and initiates the problem-solving procedure. The message generator generates appropriate outgoing messages to other agents and relevant humans. Message Controller transmits or receives the encrypted messages through TCP/IP or $X .25$ protocols using an appropriate security protocol, as will be explained further on. 


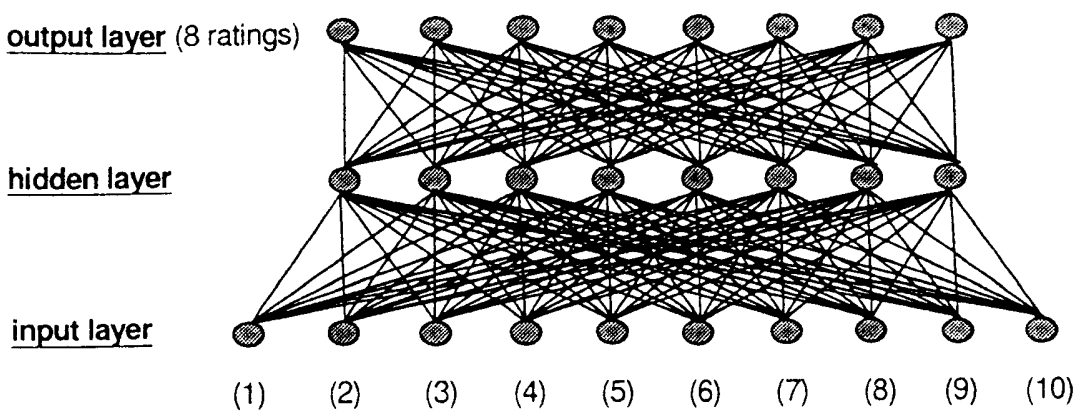

Input Factors:

(1) average balance of saving accounts in the most recent three months

(2) holding period of saving account

(3) kind of credit card possessed

(4) period of wage or pension transfer to the saving account

(5) yearly amount of public charges from saving account

(6) yearly amount of property tax in the recent year

(7) length of time working at current job

(8) current-year monthly wage or pension

(9) applicant age

(10) marital status, number of dependents

Output Factor: Customer's credit level

Figure 5. A Neural Network Model for Credit Scoring

\section{Dynamically Adjustable Credit Levels}

The checkbook agent prevents the issuance of risky checks according to the rules in the agent. However, the rules are supposed to be updated by the models in the bank's control agent. The rules include not only the level of the overdraft limit, but also the type of services allowed as determined by the level of credit. A SafeCheck checking account may have one of three modalities, all of which can be adjusted dynamically depending upon the credit level.

(1) Customers in the highest credit category (e.g., "AAA," "AA," or " $\mathrm{A}$ " in the Standard \& Poor's ratings [42]) will be qualified to issue overdrafts. This implies that authorizations will be asked only for each checkbook, not for each check. This procedure is the same as that of regular check. During the check issuance of an allowed checkbook, only extraordinary issuance may be blocked by the rules in the checkbook agent. The overdraft limit may be adjusted depending upon the credit level within this category. The fee for this service will be much lower than for credit cards or electronic fund transfer.

(2) If the customer's credit level is not high enough to qualify for the checkbook, authorization is required for each check issuance. In this sense, the procedure is the same as that of credit card, and the fee may 
Origin or

destination

Check- - Confirm legitimacy of issuing check

book - If check is legitimate, issue a check

Agent

- Otherwise, hold issuance and report to bank's control agent

- If credit level is not high enough, request authorization for each issuance

- Receive message about rule-based model

- Keep record of issued checks

Check - Check legitimacy of received

receipt checks and return receipt

agent

- Present paid checks

- Receive message about rule-based model

- Keep record of paid checks

Bank Decide registration of checking

control account and inform result

agent

- Credit scoring of checking account

- Decide issuance limit of each account and notify

- Change checking account modality with new digital certificate or suspend account

- Decide number of blank check sheets for checkbook and send them

- Urgent blocking of check issuance

-Authorize issuance for lower-credit customer

- Update account balances for cleared checks and report periodically including nonelectronic withdrawals

- Send messages about rule modification

- Send digital certificates to customer agents
Rule-based checking

Generate outgoing check message Check-receipt agent

Generate message explaining that Bank control check cannot be issued and send agent it with record of issued checks

Generate outgoing check message Bank control

for issuance authorization

agent

Modify rule-based model

Bank control

agent

Append or update to internal database

Bank control

Rule-based checking and receipt

generation

agent

Checkbook

agent

Generate outgoing message for check deposit

Modify rule-based model

Bank control agent

Bank control agent

Append or update to internal database

Bank control

agent

Rule-based decision with

Checkbook

appraisal formula and generation of message with digital certificate Neural network-based credit scoring

agent

Checkbook agent

Numerical function and message generation

Checkbook

agent

Rule-based changing of checking account modality

Checkbook

agent

Formula and message generation

Checkbook

agent

Checkbook

Generate outgoing message according to alert from user or other systems

agent

Rule-based authorization using bank's database

Checkbook agent

Clearing process with other systems

Checkbook agent, checkreceipt agent

Generate outgoing messages for updating rules

Generate outgoing message of digital certificate
Checkbook agent, checkreceipt agent Checkbook agent, checkreceipt agent

\section{Table 2. Solution Engines for SafeCheck Agents.}


be adjusted to the level of credit card service. A difference from the conventional credit card system is that the fee is charged to the check issuer, not the check recipient.

(3) If the customer's credit level is not high enough to qualify for creditbased issuance with a check-level authorization, checks can be issued only if there is a positive balance in the account, and the amount of the check cannot exceed the balance. In this sense, the check is the same as a debit card (actually the same as electronic fund transfer) or certified check whose ground is a positive balance. This service may not have a transaction cost higher than the credit card service, but a higher fee may be charged as a penalty for low credit to motivate the recovery of credit.

These dynamically adjustable service levels make SafeCheck a flexible, adaptive payment method that seeks minimal payment transaction costs and maximal credit allowances without shifting the default risk to payees.

\section{Effectiveness of the SafeCheck System}

Check systems can reduce the cost of authorization at the potential risk of default. Regular checks tolerate the risk at the cost of waived authorization costs, while credit cards reduce the risk at the cost of the authorization process. In this context, it is useful to compare the performance of three kinds of creditbased payment systems (credit-card type, check type, and SafeCheck System) in terms of the total cost of both authorization and default. The default risk by missed authorization should be distinguished from the default risk by wrong authorization. The risk of wrong authorization is not considered because it is out of the context of this research.

To compare the costs incurred by payment authorization and by defaults caused by missed authorization, consider the following factors: total number of payments $(N)$, communication cost per authorization $\left(C_{m}\right)$, computation cost per authorization $\left(C_{p}\right)$, cost per payment authorization $\left(C, C=C_{m}+C_{p}\right)$, average payment amount $(A)$, number of checks in a regular checkbook $\left(n_{r}{ }_{r}\right)$, average number of checks issued without consulting bank control agent $\left(n_{a}\right)$, issuer's credit level $(R=1, \ldots, 8)$, and default rate due to the missed authorization of the issuer's bank ( $\gamma_{c c}$ for credit-card-type payment system, $\gamma_{c}$ for check-type payment system, and $\gamma_{s}$ for SafeCheck System).

Since every payment requires the same authorization procedure as in the credit-card payment, $\gamma_{c}$ can be set to be zero. $\gamma_{c}$ can be estimated as the default rate of the regular paper check, because the check payment system here is an electronic version of the paper check system, which allows credit-based payment with the checkbook-level payment authorization. For the same number of issued checks, $\gamma_{s}$ should be less than $\gamma_{c}$ owing to the authorization capability of the checkbook agent. However, $\chi_{s}$ will become higher as the number of checks in one authorization increases. Thus, $\gamma_{s}$ as a function of $n_{a}$ is assumed in the following formula (1): 


$$
\begin{array}{ll}
\gamma_{a}^{\left(n_{a}\right)}=\alpha \cdot n_{a}^{\beta \cdot R} & \text { if } \alpha \cdot n_{a}^{\beta \cdot R}<1 \text { or } \\
\gamma^{\left(n_{a}\right)=1} & \text { if } \alpha n_{a}^{\beta \cdot R} \geq 1 .
\end{array}
$$

In formula (1), $\alpha$ and $\beta$ are positive numbers that can be estimated from the historical data. As the value of $R$ is set higher for the lower credit level, the default rate $\mathrm{\gamma}\left(n_{a}\right)$ increases up to 1 .

The unit cost per check issuance can be obtained by summing the unit defaulted amount with the unit authorization cost per check issuance, as shown in formula (2).

$$
\begin{aligned}
& f\left(n_{a}\right)=A \cdot \gamma\left(n_{a}\right)+C / n_{a} \\
& =A \cdot \alpha \cdot n_{a}^{\beta} \cdot R+C / n_{a}
\end{aligned}
$$

With the values of $A=\$ 100, \alpha=0.0005, \beta=0.2$ and $C=\$ 0.8$, the unit payment $\operatorname{cost} f\left(n_{a}\right)$ can have convex curves depending upon the issuer's credit level $(R=3,5$, or 7$)$, as shown in Figure 6 .

By differentiating $f\left(n_{a}\right)$ with $n_{a^{\prime}}$ the optimal value $n_{a}$ " can be obtained as (3):

$$
n_{a}^{*}=[C /(A \cdot \alpha \cdot \beta \cdot R)]^{1 /(1+\beta \cdot R)}
$$

With the above $n_{a}$, the bank's control agent can determine the optimal number of blank checks depending upon the issuer's credit level. In Figure 6, it can be seen that $n_{a}$ decreases as the credit level becomes lower ( $R$ becomes higher). Table 3 also shows the sensitivity of $n_{a}{ }^{*}$ on the cost per payment $(C)$ and the credit level $(R)$ of the issuer.

Table 4 compares the costs of authorization and default for three creditbased payment systems in the case where $C=\$ 0.8$ with a higher credit level of $R=3$. SafeCheck is the most economical. When a customer has a low credit level (i.e., a high default risk), and thus the optimal number of checks $\left(n_{a}\right)$ is closer to 1 , the modality of the checking account should be adjusted to credit-card or debit-card type in accordance with the bank's managerial policy.

\section{Messages Among SafeCheck Agents}

\section{Message Composition}

Conventionally, Financial Electronic Data Interchange (FEDI) can be used to exchange messages for authorization, receipt of payment, and documents for clearing [14]. However FEDI is not yet sufficiently commercialized on the Internet, and Interactive EDI [21] for independent payment participants in the open network is in the embryonic stage. FEDI seeks to attain efficiency and compatibility of communication. In the meantime, the agent community has developed 


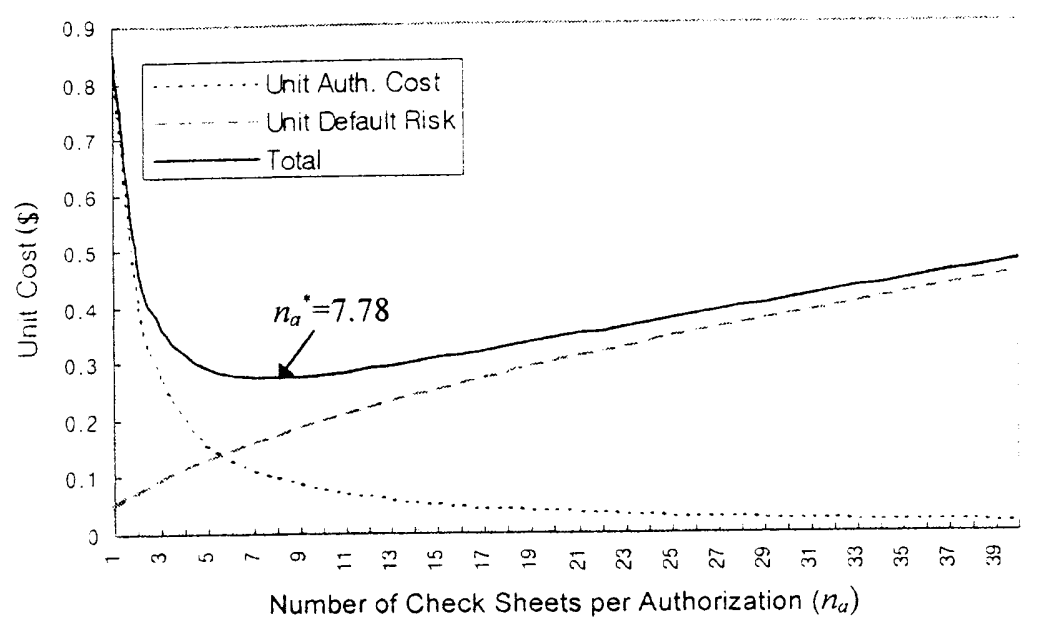

(a) Curves when an issuer's credit level $R=3$ (Relatively High)

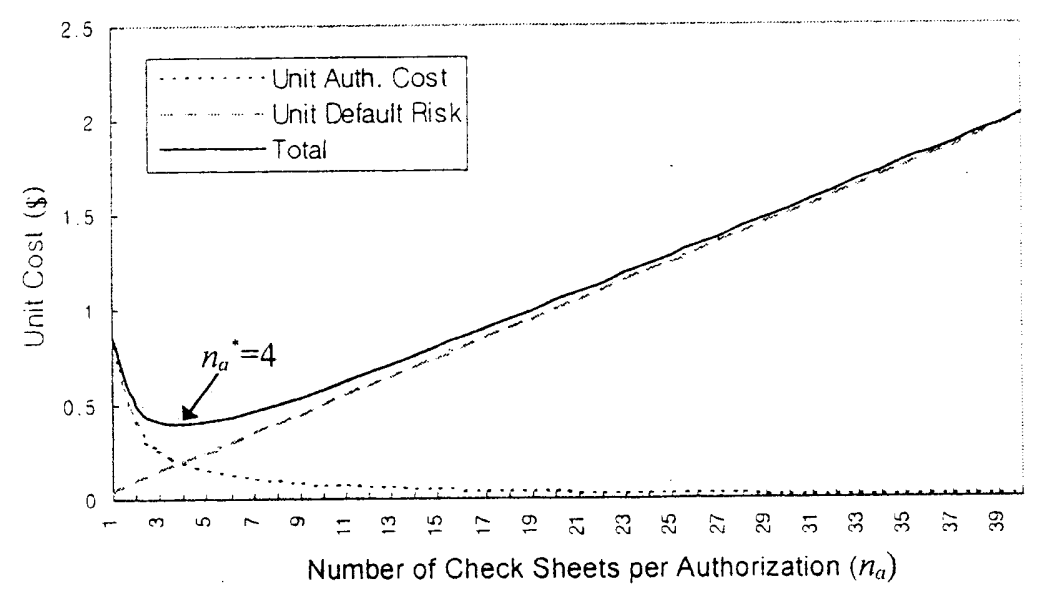

(b) Curves when an issuer's credit level $R=5$ (Relatively Medium)

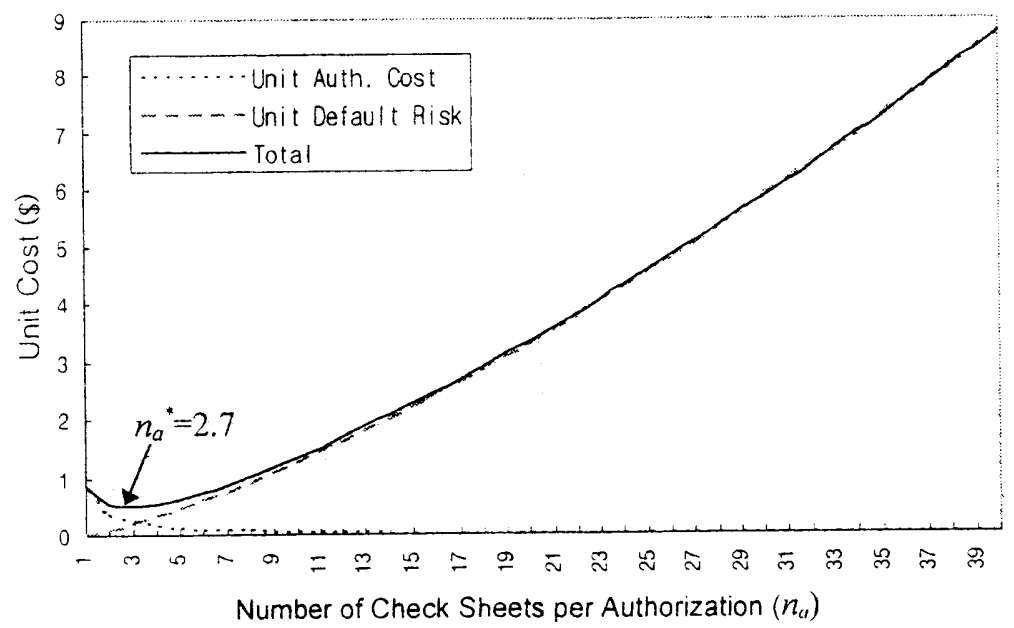

(c) Curves when an issuer's credit level $R=7$ (Relatively Low)

Figure 6. Payment Cost Curves When $A=\$ 100, \alpha=0.0005, \beta=0.2$ and $C=\$ 0.8$ 


\begin{tabular}{|c|c|c|c|c|}
\hline$c$ & $R$ & $n_{a}$ & $A \cdot \gamma\left(n_{0}\right)$ & $c / n_{0} \cdot$ \\
\hline \multirow[t]{3}{*}{$\$ 0.6$} & 3 & 6.50 & $\$ 0.154$ & $\$ 0.092$ \\
\hline & 5 & 3.46 & $\$ 0.173$ & $\$ 0.173$ \\
\hline & 7 & 2.45 & $\$ 0.175$ & $\$ 0.245$ \\
\hline \multirow[t]{3}{*}{$\$ 0.8$} & 3 & 7.78 & $\$ 0.171$ & $\$ 0.103$ \\
\hline & 5 & 4.00 & $\$ 0.200$ & $\$ 0.200$ \\
\hline & 7 & 2.76 & $\$ 0.207$ & $\$ 0.290$ \\
\hline \multirow[t]{3}{*}{$\$ 1.0$} & 3 & 8.94 & $\$ 0.186$ & $\$ 0.112$ \\
\hline & 5 & 4.47 & $\$ 0.224$ & $\$ 0.224$ \\
\hline & 7 & 3.03 & $\$ 0.236$ & $\$ 0.330$ \\
\hline \multirow[t]{3}{*}{$\$ 1.2$} & 3 & 10.03 & $\$ 0.199$ & $\$ 0.120$ \\
\hline & 5 & 4.90 & $\$ 0.245$ & $\$ 0.245$ \\
\hline & 7 & 3.27 & $\$ 0.262$ & $\$ 0.367$ \\
\hline
\end{tabular}

Table 3. Sensitivity of $n^{\circ}$ When $A=\$ 100, \alpha=0.0005$, and $\beta=0.2$.

Type Cost

Case* (million\$/year)

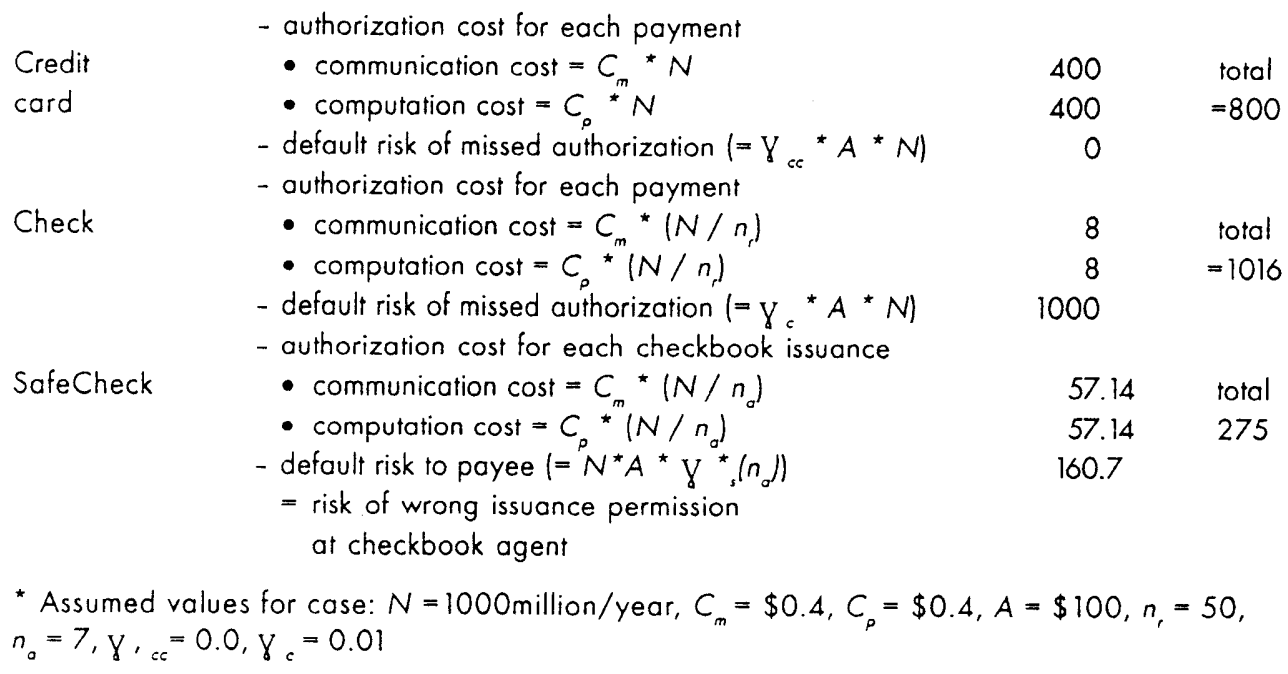

\section{Table 4. Payment Authorization Cost and Default Risk.}

agent communication languages like KQML (Knowledge Query and Manipulation Language) that pursue a richer expressiveness. Whereas the agent communication language can be regarded as a frame for logical expressions between agents, FEDI is a format for efficient physical transmission. In this sense, the FEDI engine needs to be equipped with the capability to convert the KQML message to FEDI. However, in the research stage, KQML messages can be exchanged without conversion. Instead, the security aspect must be considered.

Security is ensured by adopting the schemes used in the SET protocol [28]: public key cryptography, digital signatures, and certificates [39]. In this context, the bank's control agent plays the role of Certificate Authority (CA) for payers and payees, and the certificates are stored in the checkbook and checkreceipt agents. Upper-level CA may endorse the banks. If the designation SETChecks is applied to checks that adopt the SET-like security schemes, then in this respect SafeCheck is a SET-Check. 
In the SafeCheck System, KQML is the outer language, and messages are composed of three layers specific to the electronic check system: Agent Communication Language (ACL), Security Layer, and Check Layer (see Figure 7). The $A C L$ Layer consists of the performative and its parameters. They are independent of domain. To prevent wiretaps, the message IDs in the parameters of ":inreply-to" and ":reply-with" are encrypted with the receiver's public key.

The Security Layer is arranged in the parameter content to include the features for SET-Check as follows:

$$
\begin{array}{ll}
\text { (message } & <\text { encrypted-message-content }>\text { ) } \\
\text { (key } & <\text { encrypted-key }>\text { ) } \\
\text { (certificate } & <\text { own-certificate }><C \text { A-certificate }>\text { ) } \\
\text { (signature } & <\text { digital-signature }>\text { ) }
\end{array}
$$

The <encrypted-message-content $>$ in the message statement includes the message in the Check Layer encrypted by a symmetric algorithm like DES. The component "key" contains a symmetric key encrypted by the RSA (RavistShamir-Adelman) algorithm. The "certificate" carries the certificates of the sender and its certificate authority. The <digital-signature $>$ is an encrypted hash of the message in the Check Layer, and uses a hash algorithm, such as MD5 [39], and the sender's private key.

The Check Layer defines items specific to checks, such as requests to open checking accounts and check payments. The messages for electronic check issuance include the components depicted in Figure 7. An illustrative three-layered message for check issuance is demonstrated in Figure 8.

\section{Message Exchange and Security}

SafeCheck Agents exchange messages in KQML form through the TCP/IP communication protocol. Two performatives, such as "Evaluate" and "Reply," are adopted. "Evaluate" is used by the sending agent, and "Reply" is used by the receiving agent to confirm receipt of the messages.

Security requirements like integrity, confidentiality, and authentication are satisfied by the Security Layer. Nonrepudiation is guaranteed by keeping the sender's digital signature and received message. In particular, to identify the origin of checks and prevent forgeries, the Check Layer has "Check-ID," which contains a concatenation of the check identification encrypted with the public key and the digital signature of the check issuer's bank.

\section{Illustrative Prototype System}

This section shows how an illustrative prototype system can be implemented. Four SafeCheck Agents are implemented in four different computers to simulate the following typical transactions:

- Opening (registration) of the payer's checking account 


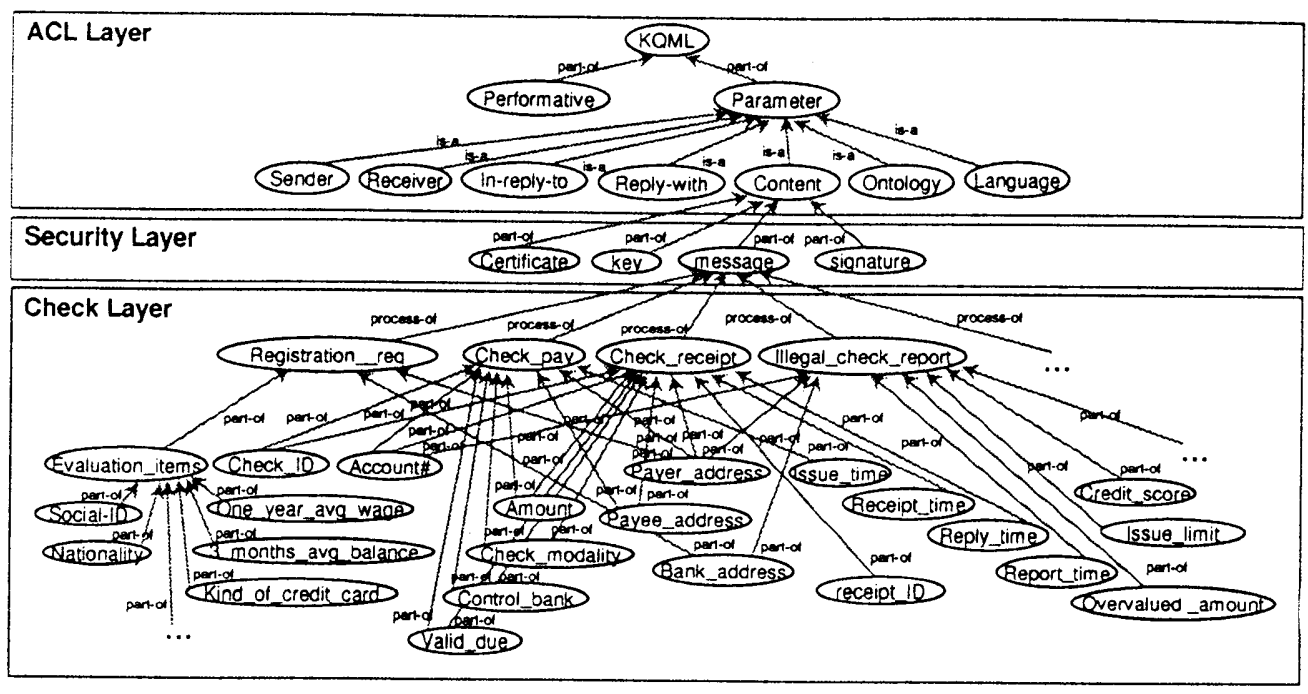

Figure 7. Message Layers in SafeCheck

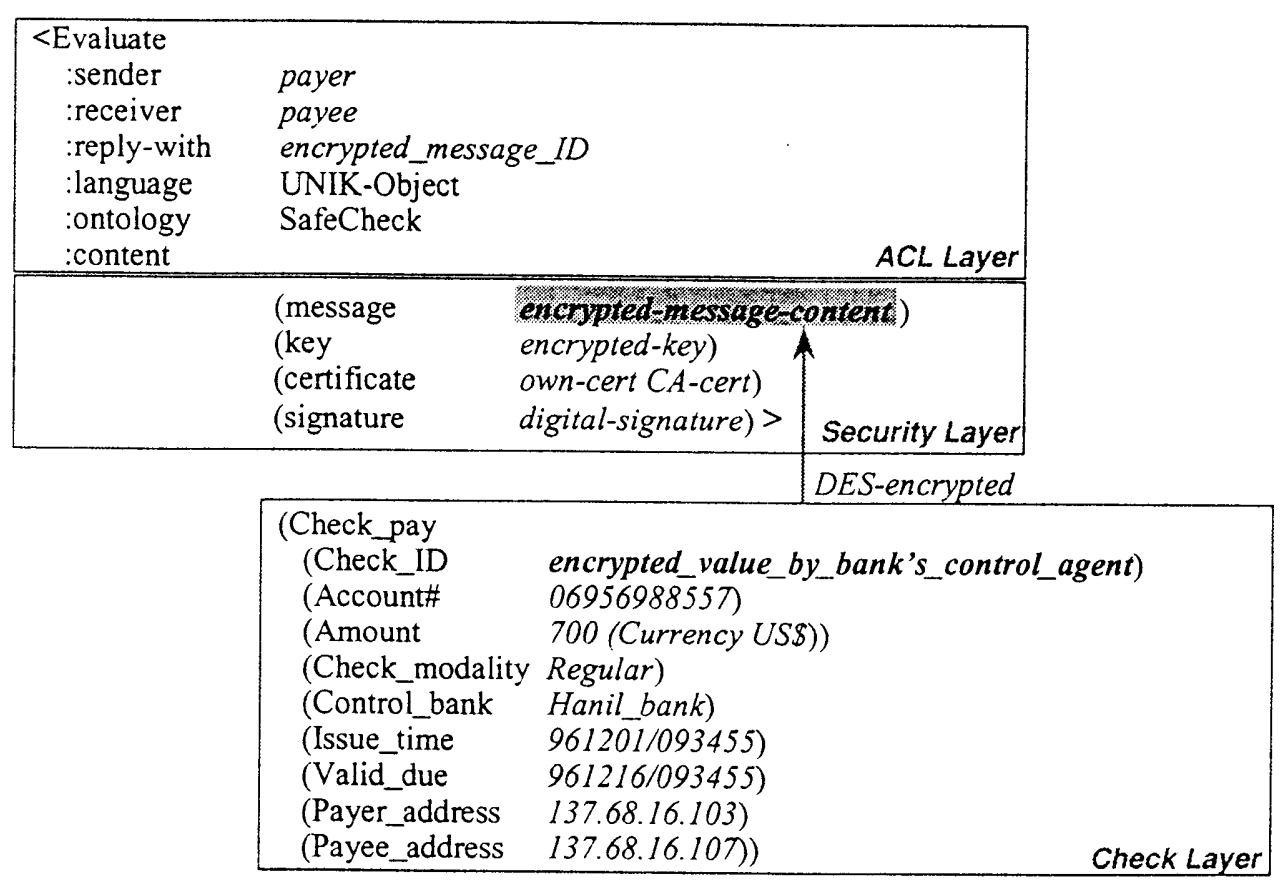

\section{Figure 8. An Example of Message for Check Issuance}

- Electronic check sheet issuance

- Payment authorization (for checkbook or check depending on the credit level)

- Other (granting of blank checks, updating of rule base in checkbook agent, paid check presentation, etc.)

The computers storing SafeCheck Agents are connected with one another through the Internet, as shown in Figure 9. In the prototype system, the clearing of presented checks from the check-receipt agent is carried out directly on-line 


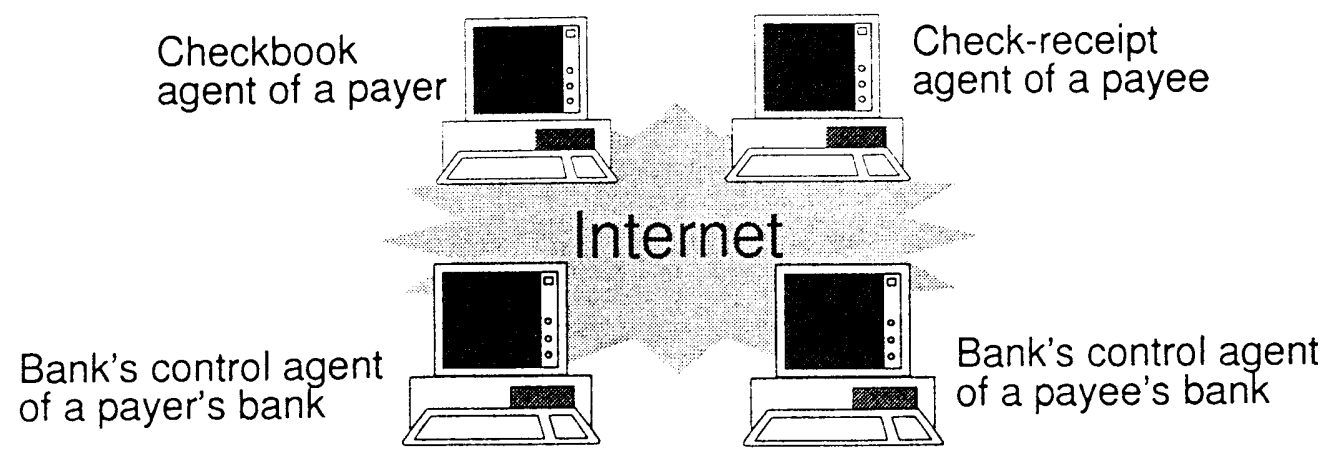

Figure 9. Architecture of the Prototype System

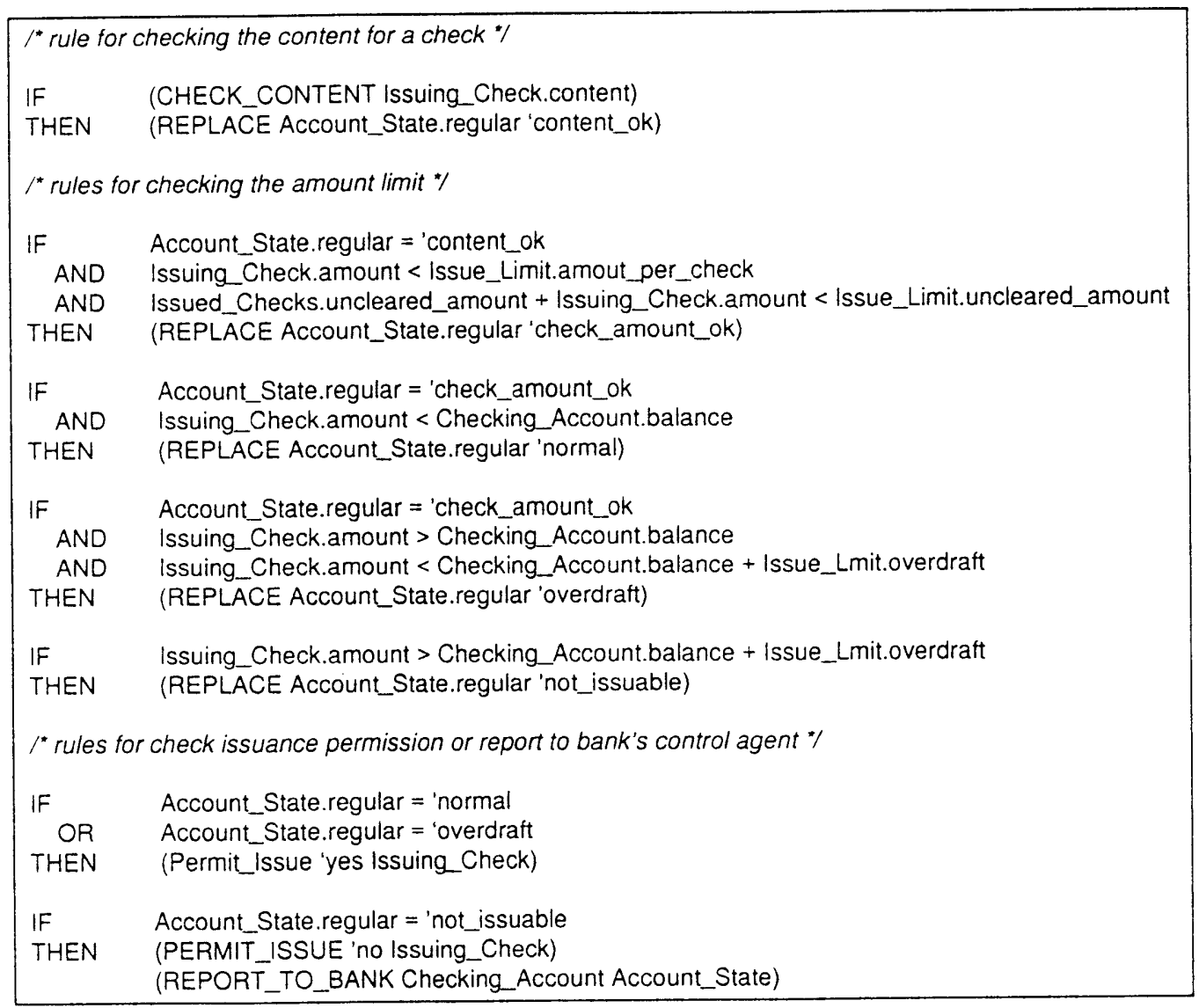

\section{Figure 10. Illustrative Rules for the Permission of Check Issuance}

between the control agents of the two banks. With $\mathrm{C}++$ language, UNIK Library is used to develop the prototype system [23]. While the checkbook agent and check-receipt agent are developed in Pentium PCs, the banks' control agents are developed in the UNIX environment of SUN workstation.

The typical solution engines of SafeCheck Agents are a forward-chaining rule-based system for the permission of check issuance, and a neural network for credit scoring. The rules on check issuance permission and issuance amount limit (see Figure 10) are implemented using UNIK-FWD [23]. The credit-scoring neural network model (see Figure 5) is implemented using UNIK-NEURO [25]. 


\section{Conclusion}

This paper proposes a virtually defaultless electronic checking system that uses three kinds of intelligent agents: a checkbook agent at the check issuer's site, a check-receipt agent at the check receiver's site, and bank control agents at the check issuer's and receiver's banks. The checkbook agent's autonomous monitoring capability prevents the issuance of bad checks. The bank's control agent adjusts the rules in the checkbook agent in accordance with the customer's credit level.

Members of the top credit class require authorization only for each checkbook with the complement of distributed monitoring. Since overdrafts are allowed, and the fee for payment for this class is lower than for credit cards, this service is appropriate for business-to-business electronic commerce. Members of the second-level credit class, as with a credit card, have to get authorization for each check, and members of the third-level credit class are allowed to issue checks only within the balance in their checking accounts. The customer's credit level is dynamically adjusted by the neural network model in the bank's control agent.

The schemes used in the SET protocol are adopted to securely implement the SafeCheck System. The system can be most safely deployed by storing the essence of the checkbook agent in the IC card. Since the SafeCheck System will prevent bad defaults, commercial banks can utilize it without imposing any risk of default on the payee's side. A minor default loss may be charged to lower-credit payers as a penalty fee, or may be absorbed by the increased revenues owing to the payee's preference.

\section{REFERENCES}

1. Benachenhou, D.; Cader, M.; and Deboeck, G. Implementation of a neural trading system. In Proceedings of International Joint Conference on Neural Networks '92. Beijing, 1992, vol. 1, pp.360-365.

2. Brown, C.; O'Leary, D.E.; and Sangster, A. AI on the WWW: Supply and Demand Agents IEEE Expert 10 (August 1995), 50-55.

3. Carter, C., and Catlett, J. Assessing credit card applications using machine learning. IEEE Expert (fall 1987), 71-79.

4. C.M.U.'s I.N.I. The NetBill project. 1997 (www.ini.cmu.edu/netbill).

5. Crede, A. Electronic commerce and the banking industry: The requirement and opportunities for new payment systems using the Internet. 1997 (jcmc.huji.ac.il/vol1/issue3/crede.html).

6. CyberCash, Inc. CyberCash-Information. 1997 (www.cybercash.com/ cybercash/news).

7. CyberCash, Inc. Introducing CyberCoin ${ }^{\mathrm{TM}} .1997$ (www.cybercash.com/ cybercash/shoppers/coingenpage.html).

8. CyberCash, Inc. PayNow ${ }^{\mathrm{TM}}$ pilot programs. 1997 (www.cybercash.com/ cybercash/paynow).

9. DigiCash. DigiCash products-the CAFE project. 1997 (www.digicash. com/products/projects/cafe.html). 
10. DigiCash. DigiCash_Publications. 1996 (www.digicash.com/publish/ publish.html).

11. Europay International. Europay profile. 1997 (www.europay.com/ prof02.htm).

12. Finin, T., Wiederhold, G.; Genesereth, M.; Fritzson, R.; McGuire, J.; Shapiro, S.; and Beck, C. Agent-Communication Agent-communication language plus example agent policies and architectures. 1996 (www.cs.umbc. edu/kqml/kqmlspec/spec.html).

13. First Vitual Holdings. The First Virtual Solution. 1997 (www.fv.com/demo).

14. Fisher Center for Information Technology and Management. Financial EDI over the Internet: A case study. Working paper WP-95-1006, 1996.

15. Forrester Research. 1997 (www.forrester.com).

16. Franklin, S., and Graesser, A. Is it an agent, or just a program?: A taxonomy for autonomous agents. 1996 (www.msci.memphis.edu/ franklin/ AgentProg.html).

17. Financial Service Technology Consortium (FSTC). FSTC electronic check project. 1996 (www.fstc.org/projects/echeck/index.html).

18. Gilbert, D.M.; Aparicio, M.; Atkinson, B.; Brady, S.; Ciccarino, J.; Grosof, B.; O'Connor, P.; Osisek, D.; Pritko, S.; Spagna, R.; and Wilson, L. The role of intelligent agents. The role of intelligent agents in the information infrastructure. Research Triangle Park, NC, IBM Co., 1995.

19. Green Sheet Online. GreenSheet annual survey. 1997 (multiplex.com/ Greensheet).

20. ISO/IEC DIS 13888-3. Information technology - Security technique-Nonrepudiation-Part 3, 1997.

21. ISO 9735-3. Electronic data interchange for administration, commerce and transport (EDIFACT)-Application level syntax rules-Part 3, 1996.

22. Japan-Singapore AI Center. PIPEMart-An electronic marketplace using intelligent agents. AI Post, Japan-Singapore AI Center Newsletter, 4, 4 (December 1996).

23. KAIST. UNIK user's manual. Seoul: Intelligent Information System Laboratory, Graduate School of Management, Korea Advanced Institute of Science and Technology, 1994 (in Korean).

24. Kalakota, R., and Whinston, A.B. Frontiers of Electronic Commerce, Reading, MA: Addison-Wesley, 1996.

25. Kim, W., and Lee, J.K. UNIK-OPT/NN: Neural network based adaptive optimal controller on optimization models. Decision Support Systems, 18 (1996), 43-62.

26. Kukmin Bank, Checking Account Procedures. 1995. Seoul: Kukmin Bank Publisher (in Korean).

27. Lee, J.K., and Lee, W. Intelligent agent based contract process in electronic commerce: UNIK-AGENT approach. In Proceedings of Hawaii International Conference on System Sciences '97. Hawaii, 1997, vol. 4, pp. 230-241.

28. MasterCard/Visa. Secure electronic transaction (SET) Specification (Version 1.0). May 1997.

29. Medvinsky, G., and Neuman, B.C. NetCash: A design for practical electronic currency on the Internet. In Proceedings of First ACM Conference on Computer and Communications Security, 1993 (www.isi.edu). 
30. Mondex International. Mondex Technology. 1996. (www.mondex.com). 31. Net1 Inc. NetChex homepage. 1996 (www.netchex.com).

32. Netscape. SSL 3.0 specification. 1996 (home.netscape.com/eng/ssl3/ index.html).

33. Network Wizard. Internet domain survey. 1997 (www.nw.com/zone/ WWW/top.html).

34. Neuman, B.C., and Medvinsky, G. Requirement for network payment: The Netcheque ${ }^{\mathrm{TM}}$ perspective. In Proceedings of IEEE Compcon' 95 . San Francisco, March 1995 (www.isi.edu).

35. Nissen, M. Intelligent agents: A technology and business application analysis. 1995 (haas.berkeley.edu/ heilmann/agents).

36. Odom, M.D., and Sharda, R. A neural network model for bankruptcy prediction. In Proceedings of IJCNN'90. San Diego, 1990, vol. 2, pp. 163-168.

37. Personal Agents. The future of electronic commerce. 1996 (www. yourcommand.com).

38. Sangster, A. The Bank of Scotland's lending adviser expert system: COMPASS. In Proceedings of the Eleventh International Conference on AIfor Applications, 1995, pp. 24-30.

39. Schneier, B. Applied Cryptography. New York: John Wiley, 1996.

40. Security First Network Bank. Bank Demonstration. 1997 (www.sfnb.com/ demos/bankdemos.html).

41. Srinivasan, V., and Kim, Y.H. Designing expert financial systems: A case study of corporate credit management. Financial Management (autumn 1988), 23-31.

42. Standard \& Poor's. Public finance issue credit ratings definitions. 1997 (www.ratings.standardpoor.com/publicfinance/pfissue.htm).

43. Surkan, A.J., and Singleton, J.C. Neural networks for bond rating improved by multiple hidden layer. In Proceedings of IJCNN ‘90. San Diego, 1990, vol. 2, pp. 157-162.

44. Tsvetovattyy, M.B.; Gini, M.; Mobasher, B.; and Wieckowski, Z. MAGMA: An agent-based virtual market for electronic commerce. Applied Artificial Intelligence 11, 6 (1997), pp. 501-523 (www.cs.umn.edu/ gini/agent. html). 45. Thirunavukkarasy, C.; Finin, T.; and Mayfield, J. Secret agents-A security architecture for the KQML agent communication language. CIKM'95 Intelligent Information Agents Workshop, Baltimore, December 1995 (www.cs.umbc. edu).

46. Utans, J., and Moody, J. Selecting neural network architecture via the prediction risk: Application to corporate bond rating prediction. In Proceedings of the First International Conference on AI Applications on Wall Street. Los Alamitos, CA: IEEE Computer Society Press, 1991, pp. 35-41.

47. Virdhagriswaran, S. Mobile unstructured Business ObjecT (MuBot) ${ }^{\mathrm{TM}}$ technology. 1996 (www.crystaliz.com/logicware/mubot.html). 48. Visa International. Visa_Visa Cash. 1997 (www.visa.com/cgi-bin/vee/ $\mathrm{nt} / \mathrm{cash} /$ main.html?2+0).

JAE KYU LEE (jklee@msd.kaist.ac.kr) is a professor in the Graduate School of Management at the Korea Advanced Institute of Science and Technology. He received his B.A. from Seoul National University, his M.S. from the Korea Advanced Institute of Science 
and Technology, and his Ph.D. from the Wharton School, University of Pennsylvania. $\mathrm{He}$ is also the director of the International Center of Electronic Commerce. He has published numerous papers in Management Science, Decision Support Systems, Expert Systems with Applications: An International Journal, Decisions Sciences, Fuzzy Sets and Systems, and International Journal of Man-Machine Studies. Currently, he is an editorial member of Decision Support Systems, Expert Systems with Applications: An International Journal, International Journal of Intelligent Systems in Accounting, Finance and Management, New Review of Applied Expert Systems, and International Journal of Electronic Commerce.

HAN SEONG YOON (yhs@msd.kaist.ac.kr) is a Ph.D. candidate in the Graduate School of Management at the Korea Advanced Institute of Science and Technology. He received a B.A. from Seoul National University and M.S. from the Korea Advanced Institute of Science and Technology. His research interests include decision-support systems, system development for electronic commerce, and integration of expert systems and optimization. 